\title{
Quasiparticle energy spectrum in ferromagnetic Josephson weak links
}

\author{
L. Dobrosavljević-Grujić, ${ }^{1}$ R. Zikić, ${ }^{1}$ and Z. Radović ${ }^{2}$ \\ November 14, 2018 \\ ${ }^{1}$ Institute of Physics, P. O. Box 57, 11080, Belgrade, Yugoslavia \\ ${ }^{2}$ Department of Physics, University of Belgrade, P.O. Box 368, 11001 Belgrade, Yugoslavia
}

\begin{abstract}
The quasiparticles energy spectrum in clean ferromagnetic weak links between conventional superconductors is calculated. Large peaks in DOS, due to a special case of Andreev reflection at the ferromagnetic barrier, correspond to spin-splitt bound states. Their energies are obtained as a function of the barrier thickness, exchange field strength, and of the macroscopic phase difference $\phi$ at the link, related to the Josephson current. In the ground state, $\phi$ can be 0 or $\pi$, depending on the ferromagnetic barrier influence. Conditions for the appearence of the zero-energy bound states (ZES) and for the spin polarized ground state (SPGS) are obtained analytically. It is shown that ZES appear only outside the weak link ground state.
\end{abstract}

PACS number: $74.50+\mathrm{r}$

Keywords: Density of states, $\pi$-contact, zero energy bound states, spin polarized ground states. Corresponding author:

L. Dobrosavljević-Grujić, Institute of Physics, P. O. Box 57, 11080, Belgrade, Serbia;

e-mail: dobrosav@phy.bg.ac.yu; zikic@ami-net.com

phone: +381 11 3162758; fax: +381 3162190 


\section{Introduction}

Recently, the study of $\pi$-junctions with an intrinsic phase difference $\phi=\pi$ in the ground state has gained much interest. Many experiments on high- $\mathrm{T}_{c}$ superconducting weak links have been interpreted in terms of $\pi$-junctions proposed for the superconductors with d-wave pairing [1]. For conventional superconductors with $s$-wave pairing, $\pi$-junctions have been suggested by Bulaevskii et al. [2] to arise in presence of magnetic impurities in the barrier, in connection with spin flip assisted coherent tunneling. Also, $\pi$-coupling was predicted theoretically to exist in superconductor-ferromagnet $(S / F)$ multilayers by Radović et al. [3]. The evidence for $\pi$ - coupling, manifested via characteristic oscillations of the superconducting critical temperature $T_{c}$ with magnetic layer thickness, was sought experimentally in several superconductorferromagnet [4, 5, 6] and superconductor-spin glass systems [7].

Other manifestations of nontrivial coupling $(\phi \neq 0)$ could be found in the density of states (DOS) and, in particular, in the appearence of the Andreev bound states at the Fermi level. Similarly as in high- $T_{c}$ superconductors, these zero-energy states (ZES) should be manifested as the zero-bias conductance peaks (ZBCP) in tunneling spectroscopy measurements [8]. Recently, Andreev reflection has been studied theoretically in $S / F$ tunnel junctions by de Jong and Beenakker [9], in $S / F / S$ junctions by Kuplevakhskii and Fal'ko [10] and by Tanaka and Kashiwaya [1], and in atomic-scale $S / F$ superlattices by Prokić and al. [12]. Recent experiments [13, 14] have shown that Andreev reflection can be strongly suppressed by spin polarization in the ferromagnet. This is the case for point contacts with transparent barriers, whereas the spin polarization can enhance the Andreev reflection in the $S / F / S$ junctions with sizeable interface scattering [15].

In the previous papers we calculated DOS and spontaneous currents in Josephson $S / F / S$ point contacts [16, 17], considering the combined effect of $d$ - wave pairing in $S$ electrodes and of the exchange field in $F$ barrier.

In the present paper we calculate the quasiparticle density of states in conventional superconductor Josephson weak links with a transparent ferromagnetic metal barrier. The results are simpler than in the general anisotropic case [16], due to the isotropy of the order parameter in $S$. The phase difference $\phi$ at the link, related to the flow of the supercurrent, in presence of the exchange energy $h$ in $F$ acquires an additional, "magnetic" contribution. We study the change in the quasiparticle energy spectrum induced by $\phi$ and $h$ and discuss the appearence of ZES and SPGS.

The paper is organized as follows: in Section II we present briefly the results of the quasiclassical theory of superconductivity for $S / F / S$ weak links: the quasiparticles Green's functions in the barrier, the resulting densities of states and the Josephson supercurrent. We show how the local densities of states can be expressed in terms of energies of bound states and give the equations for the bound states, from which follow the conditions for ZES and SPGS. We calculate the supercurrent through the link and discuss its relationship with Andreev bound states. Section III contains a discussion of the numerical results, including the comparison with the case of the normal metal $(N)$ barrier, $h=0$, and a brief conclusion.

\section{Quasiclassical Theory}

An efficient method for calculating local spectral properties of superconductors is the quasiclassical theory of superconductivity 18. In the clean limit, and assuming that the magnetic influence on superconductivity is limited to that of the exchange energy in the barrier, one can use the Eilenberger quasiclassical equations in the presence of the exchange field [19, 17, 20]. 


\subsection{Quasiparticles Green's function}

In the previous papers we have solved the quasiclassical equations for an $S / F / S$ weak link, with a transparent, thin and short $F$ barrier of thickness $2 d$, consisting of a monodomain ferromagnetic metal with constant exchange energy $h$. Here we present the results for the conventional case of $s$-wave pairing in $S$ electrodes. Both $S$ and $F$ metals are assumed clean, with same dispersion relations and with same Fermi velocity $\mathbf{v}_{\mathbf{0}}$ (electron scattering on impurities in $S$ can be neglected if $l \gg \xi_{0}$, where $l$ is the electron mean free path and $\xi_{0}$ superconducting coherence length, and if $h \gg \hbar v_{0} / l$ in $F$ ).

Taking the $x$-axis perpendicular to the barrier (Fig. 1), we assume a step-function variation of the pair potential

$$
\Delta(x)=\Delta \Theta(-d-x)+\Delta \Theta(x-d)
$$

where $\Delta=\Delta(T)$. In principle, the pair potential should be determined self-consistently. However, this would greatly complicate the calculations without shedding much light on the exchange-interaction-induced states in the $S / F / S$ case [10, 21], which is the main subject of this paper. In the $S / N / S$ case, the validity of our model requires a weak proximity effect in $S$, as it may be the case at low $T$ and for thin and short barriers.

The quasiclassical Green's function in the barrier is

$$
g=g_{\downarrow}=\frac{\omega_{n} \cos \gamma_{n}+i \Omega_{n} \sin \gamma_{n}}{\Omega_{n} \cos \gamma_{n}+i \omega_{n} \sin \gamma_{n}}
$$

where $\omega_{n}=\pi T(2 n+1)$ are the Matsubara frequencies $\left(\hbar=k_{B}=1\right), \Omega_{n}=\sqrt{\omega_{n}^{2}+|\Delta|^{2}}$,

$$
\gamma_{n}=\frac{\phi}{2}+\frac{2 h d}{\mathrm{v}_{0} \cos \varphi}-\frac{2 i \omega_{n} d}{\mathrm{v}_{0} \cos \varphi}
$$

and $\varphi$ is the angle between the direction $\mathbf{v}_{\mathbf{0}}$ and the $x$-axis [16, 17].

Outside the barrier, $|x| \geq d$, we find

$$
g_{\sigma}^{S}\left(x, \varphi, \omega_{n}\right)=g_{\sigma}\left(\varphi, \omega_{n}\right) e^{\alpha_{n}(d \pm x)}+\frac{\omega_{n}}{\Omega_{n}}\left(1-e^{\alpha_{n}(d \pm x)}\right)
$$

where $\alpha_{n}=2 \Omega_{n} / v_{0} \cos \varphi$ and $\sigma=\uparrow, \downarrow$ denotes the spin orientations with respect to the direction of the exchange field.

For the opposite spin direction, the corresponding Green's function is obtained by changing $h \rightarrow-h$

$$
g_{\uparrow}(h)=g_{\downarrow}(-h) .
$$

\subsection{Densities of states}

The quasiparticle spectrum follows from the retarded Green's functions, obtained by the analytical continuation of $g_{\downarrow}(h)$ and $g_{\downarrow}(-h)$. With our definition of $g_{\sigma}$, the partial density of states (PDOS), which is the angle-resolved DOS, is given by

$$
N_{\sigma}(x, \varphi, E)=\lim _{\delta \rightarrow 0} \Re g_{\sigma}\left(x, \varphi, i \omega_{n} \rightarrow E+i \delta\right),
$$

where $E$ is the quasiparticle energy measured from the Fermi level. 
DOS is obtained by averaging PDOS over the angle $\varphi$, assuming spherical Fermi surface

$$
N_{\sigma}(x, E) / N_{0}=\int_{0}^{\pi / 2} d \varphi \sin \varphi \mathcal{D}(\varphi) N_{\sigma}(x, \varphi, E)
$$

where $N_{0}=m k_{F} / 2 \pi^{2}$, and $\mathcal{D}(\varphi)$ is the normalized barrier transmission probability 23 . We model the barrier with a uniform probability distribution $\mathcal{D}(\varphi)=1 / \int_{0}^{\varphi_{c}} \sin \varphi d \varphi$, within an acceptance cone of angle $2 \varphi_{c}$ about the interface normal, and zero outside the cone. The relation between PDOS in $S$ :

$$
N_{\sigma}^{S}(x, \varphi, E)=\lim _{\delta \rightarrow 0} \Re g_{\sigma}^{S}\left(x, \varphi, i \omega_{n} \rightarrow E+i \delta\right),
$$

and in the barrier, Eq. (6), is obtained using Eq. (41). For $|E|<|\Delta|$ Eq. (8) reduces to the simple relation

$$
N_{\sigma}^{S}(x, \varphi, E)=e^{\alpha(d \pm x)} N_{\sigma}(\varphi, E)
$$

where $\alpha=2 \Omega / v_{0} \cos \varphi$, and $\Omega=\sqrt{|\Delta|^{2}-E^{2}}$. The influence of the barrier is seen only in the close vicinity of the interface. Deep in $S$ we obtain, as expected, the well known bulk result

$$
N^{S}(E)=\frac{|E|}{\Omega} \Theta(|E|-|\Delta|) .
$$

Since PDOS in the electrodes and in the barrier are related, we consider in the following only the position-independent PDOS and DOS in the barrier.

Measuring all energies in units of $\Delta, \tilde{E}=E / \Delta, \tilde{\Omega}=\Omega / \Delta, \tilde{h}=h / \Delta$, for PDOS we get

$$
N_{\downarrow}(\varphi,|\tilde{E}|<1)=\frac{1}{\tilde{\Omega}} \delta(\tilde{E}+\tilde{\Omega} \cot \gamma)
$$

where $\delta$ is the Dirac delta function, and

$$
N_{\downarrow}(\varphi,|\tilde{E}|>1)=\frac{-i|\tilde{E}| \tilde{\Omega}}{\tilde{E}^{2}-\cos ^{2} \gamma},
$$

where $\gamma=\phi / 2-\tilde{d}(\tilde{E}-\tilde{h}) / \cos \varphi, \tilde{d}=2 d \Delta / v_{0}=(2 / \pi) d / \xi_{0}$, and $\xi_{0}=v_{0} / \pi \Delta$.

Bound states exist only for energies $|\tilde{E}|<1$ and satisfy the equation

$$
\tan \gamma=-\frac{\tilde{\Omega}}{\tilde{E}}
$$

corresponding to the poles in the analytical continuation of the Green's function, Eq.(2)). A more convenient form of Eq. (13) is

$$
\sin \gamma= \pm \tilde{\Omega}, \quad \cos \gamma=\mp \tilde{E}
$$

with the condition

$$
\operatorname{sign}(\tilde{E}) \sin \gamma \cos \gamma \leq 0
$$

Note that the bound states with opposite spin orientation, spin up, are given by Eq. (13) (or by Eqs. (14), (15)) with $h \rightarrow-h$. There is a spin splitting of bound states. 
Another consequence of Eq. (14) is that bound states at the Fermi level (ZES) appear for $\cos \gamma=0$. For $\varphi=0$ this gives

$$
\frac{\phi}{2} \pm \tilde{d} \tilde{h}=(2 k+1) \frac{\pi}{2}, \quad k=0, \pm 1, \pm 2, \ldots
$$

Calculating DOS for $\varphi_{c}=\pi / 2$, we find

$$
\begin{aligned}
& N_{\downarrow}(|\tilde{E}|<1) / N_{0}= \\
= & 2 \pi \tilde{d}|\tilde{E}-\tilde{h}| \sum_{n=-\infty}^{\infty} \frac{\Theta(A)}{(\phi / 2+n \pi+\arctan \tilde{\Omega} / \tilde{E})^{2}}
\end{aligned}
$$

where $A=\operatorname{sign}(\tilde{E}-\tilde{h})(\Phi+n \pi)$ and $\Phi=\arctan \Omega / \tilde{E}+\phi / 2-\tilde{d}(\tilde{E}-\tilde{h})$. Let us denote by $\tilde{E}_{i}$ the zeros of Eq. (13) for $\varphi=0$, which can be written in the form $\Phi\left(\tilde{E}_{i}\right)+n_{i} \pi=0, n_{i}=0, \pm 1, \ldots$ Then, Eq. (17) can be rewritten (see Appendix A) in the form

$$
\begin{aligned}
N_{\downarrow}(|\tilde{E}|<1) / N_{0}=2 \pi \tilde{d}|\tilde{E}-\tilde{h}|\left\{\sum_{i=1}^{m} \frac{\Theta(\tilde{E}-\tilde{h}) \Theta\left(\tilde{E}_{i}-\tilde{E}\right)+\Theta(\tilde{h}-\tilde{E}) \Theta\left(\tilde{E}-\tilde{E}_{i}\right)}{\left(\phi / 2+n_{i} \pi+\arctan \tilde{\Omega} / \tilde{E}\right)^{2}}+\right. \\
\left.+\sum_{n=-\infty}^{n^{-}} \frac{\Theta(\tilde{h}-\tilde{E})}{(\phi / 2+n \pi+\arctan \tilde{\Omega} / \tilde{E})^{2}}+\sum_{n=n^{+}}^{\infty} \frac{\Theta(\tilde{E}-\tilde{h})}{(\phi / 2+n \pi+\arctan \tilde{\Omega} / \tilde{E})^{2}}\right\},
\end{aligned}
$$

where $m$ is the total number of bound states $\tilde{E}_{i}$, and integers $n^{+}, n^{-}$are defined by the conditions $\Phi+n \pi \geq 0$ for $n \geq n^{+}$and $\Phi+n \pi \leq 0$ for $n \leq n^{-}$, respectively. Integers $n_{i} \in\left[n^{-}+1, n^{+}-1\right]$ are chosen so that $\operatorname{sign}\left(\Phi(\tilde{E})+n_{i} \pi\right)=\operatorname{sign}(\tilde{E}-\tilde{h})$.

The physical meaning of this result is the following: due to the isotropy of the order parameter the averaging over the quasiparticle propagation angle $\varphi$ results in an average over one-chanel weak links of different lengths. Therefore there is no qualitative difference between DOS and PDOS, the peaks in DOS corresponding to peaks in PDOS for $\varphi=0$ situated at the energies $\tilde{E}_{i}$.

For normal metal barrier, $\tilde{h}=0$, our result agrees with Refs. [24] and [22] with small corrections of DOS for $\tilde{E} \geq \tilde{E}_{i}$. For $\phi=0$ the total number $m$ of bound states depends on the thickness, $m=2([\tilde{d} / \pi]+1)$, where the symbol $[A]$ represents the greatest integer less than $A[22$. In this case, ZES may appear only when the phase difference $\phi$ at the link is different from zero, Eq. (16).

For ferromagnetic metal barrier, $\tilde{h} \neq 0$, the ground state phase difference $\phi_{g s}$ has been calculated [17] by minimizing the energy of the link, and it was found that $\phi_{g s}=0$ for weak barrier influence, $2 \tilde{h} \tilde{d}<1$, and $\phi_{g s}=\pi$ for stronger influence, $1<2 \tilde{h} \tilde{d}<4$. Larger values of $2 \tilde{h} \tilde{d}$ would correspond to the decoupling of $S$ electrodes [10]. Combining this result with the condition for ZES, Eq. (16), one can easily see that ZES can occur for $\phi \neq \phi_{g s}$ only. Due to the spin splitting, SPGS may appear [10, 11, 21]. Analyzing Eq. (13) for $\varphi=0$, we find that for $\tilde{d} \geq \pi / 2$ there is always at least one bound state for each spin orientation in the interval $-1<\tilde{E}<0$. Therefore, the spin polarized ground state, where only one spin orientation negative energy levels are populated, may exist only in thin barriers, $\tilde{d}<\pi / 2$. They actually appear (see Appendix B), with spin up orientation, provided that $\phi / 2+\tilde{d} \tilde{h}$ belongs to the (open) interval $((2 k+1) \pi / 2,(k+1) \pi-\tilde{d})$, whereas $\phi / 2-\tilde{d} \tilde{h}$ lies outside the above interval, and vice versa for spin down orientation.

We emphasize that all above analytical results are in complete agreement with numerical calculations of PDOS and DOS, which we performed directly from Eqs. (2) and (6). In numerical 
calculations, we have everywhere taken $\varphi_{c}=\pi / 2$. For smaller $\varphi_{c}$ there is no qualitative difference in DOS, the number and the positions of the peaks are the same, the relevant direction being that perpendicular to the barrier, $\varphi=0$. This is not the case for $d$-wave pairing in cuprates, as shown in Ref. [16].

\subsection{Supercurrents}

The supercurrent trough the $S / F / S$ junction is also obtained from the Green's functions in the barrier, Eqs. (2) and (5).

$$
\mathbf{j}=-2 i e \pi N_{0} T \sum_{\omega_{n}}\left\langle\mathbf{v}_{0} \frac{g_{\uparrow}+g_{\downarrow}}{2}\right\rangle
$$

where $\langle\cdots\rangle$ denotes the angular averaging over the Fermi surface. Putting $u=1 / \cos \varphi$ we get

$$
I=\frac{\pi I_{0} T}{4 \Delta} \sum_{\omega_{n}} \sum_{\sigma} \int_{1}^{\infty} \frac{\Im\left[g_{\sigma}(u)+g_{\sigma}(-u)\right]}{u^{3}} d u, \quad \sigma=\downarrow, \uparrow .
$$

This gives the supercurrent through the barrier of the area $S, I=j S$ as a function of $\phi$, temperature $T$, and of parameters measuring the influence of the $\mathrm{F}$ barrier, $h$ and $d$. The normalizing current is $I_{0}=2 \Delta / e R_{N}$, and the normal resistance is given by $R_{N}^{-1}=e^{2} \mathrm{v}_{0} N_{0} S$, where $N_{0}$ is the density of states at the Fermi surface.

From Eq. (19) we see that poles of $g_{\sigma}$ determine both the quasiparticle energy spectrum and the phase and exchange field dependence of the supercurrent. That the supercurrent is carried by the Andreev bound state is particularly clearly seen at $T=0$. Taking $T \sum_{\omega_{n}} \rightarrow \int d \omega / 2 \pi$, it is easy to show that Eq. (19) at zero temperature can be rewritten in the form

$$
I(\phi)=\frac{\pi}{2} I_{0} \sum_{j} \int_{1}^{\infty} \frac{d u}{u^{3}}\left[\frac{\partial \tilde{E}_{j, \downarrow}}{\partial \phi}+\frac{\partial \tilde{E}_{j, \uparrow}}{\partial \phi}\right]
$$

where $\tilde{E}_{j, \downarrow}$ and $\tilde{E}_{j, \uparrow}$ are negative energy levels, obtained from Eq. (14) with $\gamma=\gamma( \pm h)$. Since the pair potential is assumed to be spatially constant in the $S$ electrodes, the free energy of the junction is related to the supercurrent by the well-known relation

$$
I(\phi)=2 e \frac{\partial F}{\partial \phi}
$$

and

$$
2 e F(\phi)=\frac{\pi}{2} I_{0} \sum_{j} \int_{1}^{\infty} \frac{d u}{u^{3}}\left[\tilde{E}_{j, \uparrow}+\tilde{E}_{j, \downarrow}\right]
$$

In particular, in the limit of strong exchange field, $\tilde{h} \gg 1$, we obtain

$$
I=\frac{\pi}{4} I_{0} \int_{1}^{\infty} \frac{d u}{u^{3}}\left[\sin \left(\frac{\phi}{2}+\tilde{d} \tilde{h} u\right) \operatorname{sign}\left(\cos \left(\frac{\phi}{2}+\tilde{d} \tilde{h} u\right)\right)+\sin \left(\frac{\phi}{2}-\tilde{d} \tilde{h} u\right) \operatorname{sign}\left(\cos \left(\frac{\phi}{2}-\tilde{d} \tilde{h} u\right)\right)\right]
$$

and

$$
2 e F(\phi)=-\frac{\pi}{2} I_{0} \int_{1}^{\infty} \frac{d u}{u^{3}}\left[\left|\cos \left(\frac{\phi}{2}+\tilde{d} \tilde{h} u\right)\right|+\left|\cos \left(\frac{\phi}{2}-\tilde{d} \tilde{h} u\right)\right|\right] .
$$

Therefore, even in this limit, the supercurrent is carried by the Andreev bound states of both spin orientations, and the phase dependence is not sinusoidal, in contrast to the results of Tanaka and Kashiwaya for ferromagnetic tunnel junctions [11. 


\section{Summary and discussion}

In this Section, we discuss the results obtained for $S / N / S$ and $S / F / S$ weak links, with thin metallic barrier, $2 d / \xi_{0}<\pi(\tilde{d}<1)$.

The results of numerical calculation of the supercurrent at $T=0$ as a function of $\phi$, for some characteristic values of $h$, are shown in Fig. 2, and the results for PDOS and DOS in Figs. 3-6. In all examples we have taken $2 d=\xi_{0}(\tilde{d}=1 / \pi)$.

In both cases of normal $(h=0)$ or ferromagnetic $(h \neq 0)$ metal barrier, the quasiparticle spectrum in the barrier is gapless, with characteristic peaks inside the superconducting gap, $|E|<\Delta$. Due to the isotropy of the order parameter in $S$, there is no qualitative difference between PDOS for $\varphi=0$ and DOS. The peaks in PDOS, corresponding to the bound states, are reflected in DOS as peaks placed at the same energies. This is illustrated for $h=0$ in Fig. 3 , but holds for $h \neq 0$ as well.

For thin normal metal barrier, the number of bound states is $m=2$ or $m=1$. The spectra are strongly influenced by the phase difference $\phi$. A single peak, corresponding to ZES, appears for $\phi=\pi$ at the maximum of the supercurrent through the link, Fig. 2(a). In the ground state, $\phi=\phi_{g s}=0$, DOS starts from zero at $E=0$ and has two peaks at $E \neq 0$, Fig. 3 .

For ferromagnetic metal barrier, $h \neq 0$, the bound states for two spin orientations (spin up and spin down with respect to the exchange field orientation) are no more degenerate. However, both kinds of bound states contribute to the supercurrent through the link. The dependence $I(\phi)$ is shown in Fig. 2(b) for $\phi_{g s}=0$ and for $\phi_{g s}=\pi$. DOS curves are splitted with respect to the case $h=0$, and the number of peaks is doubled. An example with $\phi_{g s}=0$ is shown in Fig. 4.

Now, the conditions for ZES, Eq. (16), are $\phi \pm 2 \tilde{d} h=\pi$. The main effect of $h \neq 0$ is to induce an additional, "magnetic" phase difference $\phi \rightarrow \phi \pm 2 \tilde{d} \tilde{h}$, for the electrons traveling in the direction $\varphi=0$. Thus, the formation of ZES is due to an appropriate value of the total phase difference. When a ZES appears for a given value of $\phi$ and $\tilde{h}$ for one spin direction, there is no ZES for the same $\phi$ for the opposite spin direction, $h \rightarrow-h$, except if $\phi=0$ and $\tilde{d} \tilde{h}=\pi / 2$. The latter case is illustrated in Fig. 5 for $\tilde{h}=\pi^{2} / 2$. Here $\phi_{g s}=\pi$, and ZES is obtained outside the ground state, for $\phi=0$.

Appearence of spin polarized states, with a single spin orientation at negative energies, is illustrated in Fig. 6 , for $\tilde{h}=1.5$. For given $\tilde{h}$ and $\tilde{d}$, the spin orientation of the polarized state strongly depends on the phase difference $\phi$. In the given example, the negative energy state in the ground state, $\phi=\phi_{g s}=0$, has the spin down orientation, and for $\phi=\pi$, the spin up orientation, in accordance with the condition (27) in the Appendix B.

In conclusion, we have analyzed the quasiparticle spectra in clean $S / F / S$ contacts, with $s$-wave pairing in $S$ electrodes. In this case DOS for each spin orientation is asymmetrical, and strongly exchange field and phase dependent. For both ferromagnetic and normal metal barriers we have found conditions for the formation of zero energy bound states, and in the case of ferromagnetic barrier, for the formation of spin polarized ground states. In all cases, zero energy bound states appear only outside the weak link ground state.

\section{Appendix A}

In this Appendix we show how in the case of $s$-wave pairing, DOS for $\tilde{E}<1$, Eq. (17), can be expressed in terms of bound states energies, Eq. (18). Since the function $\Phi=\arctan \Omega / \tilde{E}+$ $\phi / 2-\tilde{d}(\tilde{E}-\tilde{h})$ is bounded in the interval $|\tilde{E}|<1$, there is a minimum integer $n^{+}$such that $\Phi_{\min }+n^{+} \pi \geq 0$. This means that for each $n \geq n^{+}$and each $\tilde{E}, \Phi+n \pi \geq 0$. Similarly, there 
is a maximum integer $n^{-}$such that for each $n \leq n^{-}$, and for each $\tilde{E}, \Phi+n \pi \leq 0$. Thus, Eq. (17) can be written in the form

$$
\begin{array}{r}
N_{\downarrow}(|\tilde{E}|<1) / N_{0}=2 \pi \tilde{d}|\tilde{E}-\tilde{h}|\left\{\sum_{n=-\infty}^{n^{-}} \frac{\Theta(\tilde{h}-\tilde{E})}{(\phi / 2+n \pi+\arctan \tilde{\Omega} / \tilde{E})^{2}}+\right. \\
\left.+\sum_{n=n^{+}}^{\infty} \frac{\Theta(\tilde{E}-\tilde{h})}{(\phi / 2+n \pi+\arctan \tilde{\Omega} / \tilde{E})^{2}}+\sum_{n=n^{-}+1}^{n^{+-1}} \frac{\Theta(A)}{(\phi / 2+n \pi+\arctan \tilde{\Omega} / \tilde{E})^{2}}\right\},
\end{array}
$$

where $A=\operatorname{sign}(\tilde{E}-\tilde{h})(\Phi+n \pi)$. In this expression the first two sums are continuous functions in the whole interval $|\tilde{E}|<1$. In the third sum, all terms for which the condition $\operatorname{sign}(\Phi(\tilde{E})+n \pi)=\operatorname{sign}(\tilde{E}-\tilde{h})$ is not satisfied are missing, giving rise to finite discontinuities. These discontinuities occur at bound states energies $\tilde{E}=\tilde{E}_{i}$ for $\varphi=0$, as can be seen by considering the behavior of the function $\Phi(\tilde{E})$, which is monotonous and descending in the intervals $(-1,0)$ and $(0,1)$, with a discontinuity at $\tilde{E}=0$. In fact, $\Phi(\tilde{E})+n_{i} \pi$ changes sign in the vicinity of $\tilde{E}_{i}$, since by definition $n_{i}$ is an integer such that $\Phi\left(\tilde{E}_{i}\right)+n_{i} \pi=0$. For $\Phi(\tilde{E})+n_{i} \pi<0$ and $\tilde{E}-\tilde{h}>0$, as well as for $\Phi(\tilde{E})+n_{i} \pi>0$ and $\tilde{E}-\tilde{h}<0$, the $n_{i}^{\text {th }}$ term in the third sum in Eq. (25) is missing due to the step function $\Theta(A)$. Therefore, the sum is discontinuous at $\tilde{E}=\tilde{E}_{i}$, and the third sum over $n$ in Eq. (25) can be written as a sum over $\tilde{E}_{i}$ in Eq. (18).

\section{Appendix B}

In this Appendix we derive the conditions for the appearence of the spin polarized states from Eq. (13), written in the form $\Phi(\tilde{E})=n \pi, n=0, \pm 1, \pm 2, \ldots$, where $\Phi(\tilde{E})=\arctan \tilde{\Omega} / \tilde{E}+$ $\phi / 2-\tilde{d}(\tilde{E} \mp \tilde{h})$ for spin down (up) orientation. Let us denote the greatest number $n$ for which $\Phi(\tilde{E}=-1)>n \pi$ by $n_{0}=[\Phi(\tilde{E}=-1) / \pi]$, where the symbol $[A]$ represents the greatest integer less than $A$.

Since $\Phi(\tilde{E})$ is monotonously decreasing in the energy intervals $(-1,0)$ and $(0,1)$ (with a discontinuity at $\tilde{E}=0$ ), there would be no solution in the negative energies interval, if $\Phi\left(\tilde{E}=0^{-}\right)>n_{0} \pi$, or explicitly if

$$
\frac{\phi / 2 \pm \tilde{d} \tilde{h}-\pi}{\pi}>\left[\frac{\phi / 2 \pm \tilde{d} \tilde{h}-\tilde{d}}{\pi}\right] .
$$

One can check that the condition (26), which means that there is no spin down (up) bound states in the negative energies interval $(-1,0)$, is satisfied provided that

$$
\phi / 2 \pm \tilde{d} \tilde{h} \in((2 k+1) \pi / 2,(k+1) \pi-\tilde{d}) .
$$

Therefore, to have a spin up polarized ground state, $\phi / 2+\tilde{d} \tilde{h}$ must be inside and $\phi / 2-\tilde{d} \tilde{h}$ outside the above (open) interval, and vice versa for the spin down polarized ground state.

Similar conditions hold in the positive energies interval $(0,1)$. There is no spin down (up) states provided that

$$
\frac{\phi / 2 \pm \tilde{d} \tilde{h}-\tilde{d}}{\pi}>\left[\frac{\phi / 2 \pm \tilde{d} \tilde{h}+\pi / 2}{\pi}\right]
$$

i. e.

$$
\phi / 2 \pm \tilde{d} \tilde{h} \in(k \pi+\tilde{d},(2 k+1) \pi / 2) .
$$


For $\tilde{d}>\pi / 2$ neither of the conditions (27) nor (29) is satisfied, so there is no spin polarized states. Note that the condition for ZES is obtained when $\phi / 2 \pm \tilde{d} \tilde{h}=(2 k+1) \pi / 2$, i. e. at the only common point of two intervals in (27) and (29).

\section{References}

[1] D. J. van Harlingen, Rev. Mod. Phys. 67, 515 (1995).

[2] L. N. Bulaevskii, V. V. Kuzii, and A. A. Sobyanin, Zh. Eksp. Teor. Fiz., Pis'ma 25, 314 (1977) [JETP Lett. 25, 290 (1977)].

[3] Z. Radović, M. Ledvij, L. Dobrosavljević-Grujić, A. I. Buzdin, and J. R. Clem, Phys. Rev. B 44, 759 (1991); Z. Radović and L. DobrosavljevićGrujić, in Superconducting Superlattices and Multilayers, Proc. SPIE v. 2157, 240 (1994).

[4] C. Strunk, C. Sürgers, U. Pashen, and H. v. Löhneysen, Phys. Rev. B 49, 4053 (1994).

[5] J. S. Jiang, D. Davidović, Daniel H. Reich, and C. L. Chien, Phys. Rev. Lett. 74, 314 (1995).

[6] Y. Obi, M. Ikebe, T. Kubo, and H. Fujimori, Physica C, 317-318, 149 (1999).

[7] L. V. Mercaldo, C. Attanasio, C. Coccorese, L. Maritato, S. L. Prischepa, and M. Salvato, Phys. Rev. B 53, 14040 (1996).

[8] S. Kashiwaya and Y. Tanaka, Phys. Rev. B 51, 1350 (1995); Y. Tanaka and S. Kashiwaya, Phys. Rev. B 56, 892 (1997).

[9] M. J. M. de Jong and C. W. J. Beenakker, Phys. Rev. Lett. 74, 1657 (1995).

[10] S. V. Kuplevakhskĩ and I. I. Fal'ko, Pis'ma Zh. Eksp. Teor. Fiz. 52, 957 (1990) [JETP Lett., 52, 340 (1990)].

[11] Y. Tanaka and S. Kashiwaya, Physica C 274, 357 (1997).

[12] V. Prokić, A. I. Buzdin and L. Dobrosavljević-Grujić, Phys. Rev. B 59, 587 (1999).

[13] R. Soulen et al., Science 282, 85 (1998).

[14] S. Upadgyay, A. P. R. Louie, and R. Buhrman, Phys. Rev. Lett. 81, 3247 (1998).

[15] O. Bourgeois et al., preprint cond-mat/9901045.

[16] R. Žikić, L. Dobrosavljević-Grujić and Z. Radović, Phys. Rev. B 59, 14 644 (1999). 
[17] Z. Radović, L. Dobrosavljević-Grujić and B. Vujičić, Phys. Rev. B 60, 6844 (1999); L. Dobrosavljević-Grujić and Z. Radović, in Spectroscopic Studies of Superconductors, Proc. SPIE 2696, 428 (1996).

[18] G. Eilenberger, Z. Phys. 214, 195 (1968).

[19] A. I. Buzdin et al., Pis'ma Zh. Eksp. Teor. Fiz. 35, 147 (1982) [JETP Lett. 35, 179 (1982)].

[20] J. A. X. Alexander et al., Phys. Rev. B 31, 5811 (1985).

[21] M. J. DeWeert and G. B. Arnold, Phys. Rev. B 39, 11307 (1989).

[22] G. B. Arnold, Phys. Rev. B 18, 1076 (1978).

[23] M. Fogelstrom et al., Phys. Rev. Lett. 79, 281 (1997).

[24] P. G. de Gennes and D. Saint James, Phys. Lett. 4, 151(1963). 


\section{Figure 1:}

Schematic illustration of the weak link.

Figure 2:

Normalized supercurrent at zero temperatures for (a) $S / N / S$, and (b) $S / F / S$ weak links. In both cases the reduced thickness of the barrier is $2 d / \xi_{0}=1$. For $S / F / S$ two examples are presented: a "0-contact", $\phi_{g s}=0$, with $h / \Delta=$ 1.5 (dotted curve), and a " $\pi$-contact", $\phi_{g s}=\pi$, with $h / \Delta=4.7$ (solid curve).

Figure 3:

Densities of states in the barrier of $S / N / S$ weak link with $2 d / \xi_{0}=1$ : (a) PDOS for $\varphi=0$, (b) DOS averaged over the spherical Fermi surface for $\phi=0$ (solid curves), and for $\phi=\pi$ (dashed curves).

Figure 4:

DOS in the ferromagnetic barrier of $S / F / S$ weak link with $2 d / \xi_{0}=1$ and $h / \Delta=0.6$ : (a) ground state, $\phi=0$, (b) $\phi=\pi$. Spin splitting: $\sigma=\uparrow$ (solid curves) and $\sigma=\downarrow$ (dotted curves).

Figure 5:

DOS in the ferromagnetic barrier of $S / F / S$ weak link with $2 d / \xi_{0}=1$ and $h / \Delta=\pi^{2} / 2$ : (a) $\phi=0$, (b) ground state, $\phi=\pi$. Spin splitting: $\sigma=\uparrow$ (solid curves) and $\sigma=\downarrow$ (dotted curves). Note that for $\phi=0$ ZES appear for both spin orientations in accordance with Eq. (16)

Figure 6:

DOS in the ferromagnetic barrier of $S / F / S$ weak link with $2 d / \xi_{0}=1$ and $h / \Delta=1.5$ : (a) ground state, $\phi=0$, (b) $\phi=\pi$. Spin splitting: $\sigma=\uparrow$ (solid curves) and $\sigma=\downarrow$ (dotted curves). 
This figure "DobrosavFig1.gif" is available in "gif" format from: http://arxiv.org/ps/cond-mat/9911339v1 
This figure "DobrosavFig2.gif" is available in "gif" format from: http://arxiv.org/ps/cond-mat/9911339v1 
This figure "DobrosavFig3..gif" is available in "gif" format from: http://arxiv.org/ps/cond-mat/9911339v1 
This figure "DobrosavFig4.gif" is available in "gif" format from: http://arxiv.org/ps/cond-mat/9911339v1 
This figure "DobrosavFig5.gif" is available in "gif" format from: http://arxiv.org/ps/cond-mat/9911339v1 
This figure "DobrosavFig6.gif" is available in "gif" format from: http://arxiv.org/ps/cond-mat/9911339v1 